One statement in Prof. Reynolds's paper I must protest against; on page 147 he says of me: "showing that he has in no way realised the point of Clausius's paper which ho quotes-showing, in fact, that he cannot even have read it." I am sorry that without just cause Prof. Reynolds should disfigure a scientific discussion with an assertion such as the last part of this.

While disclaiming the errors that Prof. Reynolds would saddle me with, I must confess that the theory of my paper is far less complete than I should like to see it, but I have a partial excuse in that I cat the space devoted to the bare essentials of the theory down to a minimum, seeing that its applications made a demand on your space which, it appeared to me, might jeopardize their being published at all.

Melbourne, 19th April, 1897. Yours obediently, WiLliam SUTHerLand.

VII. Doublets and Triplets in the Spectrum produced by External Magnetic Forces. By Dr, P. ZeEman *.

1. $\mathrm{N}$ a former occasion $\dagger \mathrm{I}$ have remarked that the elementary treatment of the general idea of the Lorentz theory of the magnetic broadening of the spectral lines indicates that the broadened line must in some cases be broken up into a triplet. I have examined this subject somewhat more in detail. Further consideration shows that with a very strong magnetic field, a magnetically broadened line must be broken up into doublets or triplets according as the light is emitted in a direction parallel or perpendicular respectively to the lines of force. With a smaller intensity of the field, the line will be simply widened. The prediction of Lorentz with regard to the polarization of the edges of the broadened lines having been fulfilled, it seemed worth while to pursue still further the study of the polarization of the lines. Even with magnetic forces insufficient to break up the line into a triplet, it might be possible to learn something more about the constitution of the widened line. It seemed to me to be of interest to investigate this point, and to see whether the explanation formerly given, intended as the very first sketch of the motion of ions according to the theory of Lorentz, was further confirmed by experiment.

Prof. Oliver Lodge $\ddagger$ has suggested that, under some sup-

* Communicated by Prof. Oliver Lodge.

$\dagger$ Phil. Mag. for March 1897.

$\ddagger$ 'The Electrician' for February 28, 1897, p. 569 
positions, we might conclude also that the line will be simply widened in a strong field. I take the liberty of stating briefly to the readers of the 'Philosophical Magazine' the preliminary results I have obtained in regard to these questions.

2. In $\$ 18$ of the cited paper, the motion of an ion in a magnetic field was treated according to Lorentz's theory. The system of coordinates was chosen in such a manner that the axis of $z$ was parallel to the magnetic force, the plane of $(x, y)$ being perpendicular to it. Referring for the rest to the cited paper, I recapitulate that the considered motions of the ions, existing before the putting on of the magnetic force, were resolved into a rectilinear harmonic motion parallel to the axis of $z$ and two circular (right-handed and left-handed) motions in the plane of $(x, y)$.

The first remains unchanged under the influence of the magnetic force, the periods of the last are changed.

3. Using the rule given by Lorentz, therefore, we see that in the direction of the lines of force, right-handed and lefthanded circularly polarized light of changed period must be propagated. The ions vibrating parallel to the lines of force of course do not cause any disturbance in the æther equivalent to light in that direction.

In a direction perpendicular to the lines of force, those motions of the ions which have a component parallel to the plane of $(x, y)$ give plane-polarized light vibrating in a vertical plane and with changed period (I suppose the axis of $z$ in a horizontal plane). The ions, however, moving parallel to the axis of $z$ give electrical vibrations in a horizontal direction with unchanged period.

4. Magnetic Doublets.-Hence with every value of the magnetic force, an infinitely narrow spectral line breaks up into two lines, the light being emitted in the direction of the lines of force. However, for lines of finite width the magnetic change of the period must exceed somewhat that corresponding to half the width of the original line in order that the doublet may be seen. One of the components then must be over the whole width left-handed, the other right-handed circularly polarized.

5. Magnetic Triplets. - With finite width of the spectral line and observing in a direction perpendicular to the lines of force, the line is broken up into a triplet if the magnetic change of the period corresponds to the whole width of the original spectral line. Hence the magnetic force necessary to produce a triplet is equal to twice the value nesessary for a doublet.

6. Intermediate Forms of Magnetic Doublets and of Triplers -With magnetic forces less than the ones supposed in $\$ \$ 4$ 
and 5 , forms intermediate between the unchanged spectral lines and the doublets and triplets may be expected. With the doublet only one particular intermediate form exists, viz., a line the edges of which are cireularly polarized, the central part emitting unpolarized light. This case I described in my former paper.

Of triplets two intermediate forms may be distinguished.

I will call a line "Triplet $a$," if the magnetic change corresponds to somewhat more than half the width of the unchanged line. In this case the broadened line is composed in the following manner: the central part will emit horizontal vibrations, at both its sides bands of (chiefly, but not entirely) unpolarized light border it, which again are enclosed by bands of vertically vibrating light. The accompanying diagram gives a rough scheme of the constitution of the line.

\section{Polarized light.}

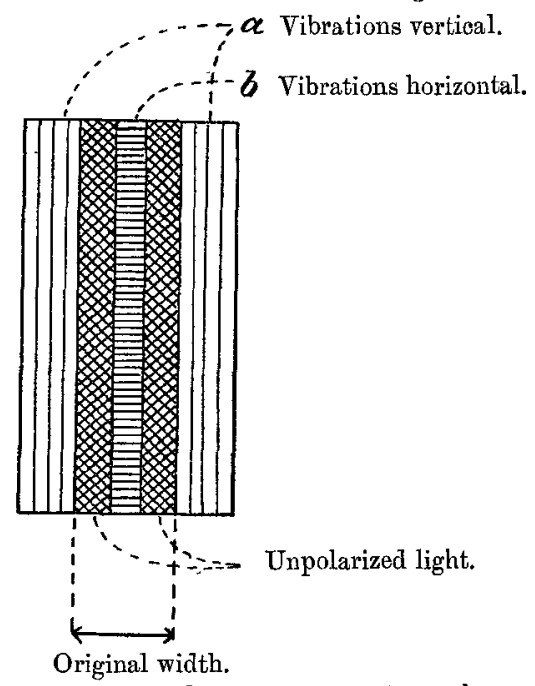

In "Triplet $b$ " the magnetic change amounts to less than half the width of the original line. This case I described in my cited paper.

7. Hence we may expect that if we can just resolve the doublet, the magnetic force also is sufficient for triplet $a$.

As with sodium $(c f . \S 11)$ I did not succeed completely with the means at my disposal in observing the expected doublets and triplets, so with other substances I looked in the first place for magnetic doublets. We might expect then that further inquiry would show something about the triplets. At last I succeeded in observing with the blue line $(\lambda=480 \mu \mu)$ of cadmium the doublet and the triplet $a$. It has already been remarked by 
Egoroff and Georgiewsky * that cadmium also exhibits the broadening of the spectral lines under the action of magnetism.

8. The method of experiment was principally the same as that formerly used. As in my former experiments, I used a Ruhmkorff electromagnet. I missed, however, the beautiful Rowland grating I used in the laboratory of Prof. Onnes. I now only had at my disposal a smaller one with a radius of $6 \mathrm{ft}$, but like the Leyden one with 14438 lines to the inch. The second spectrum was very satisfactory. For the cadmium spectrum a spark was used between cadmium electrodes; with a lens, as often used in this manner by Lockyer, an image of spark and electrodes was formed on the slit; it is then easily verified that the part of the spark just between the poles is analysed.

The above-mentioned cadmium line is especially sharp at the violet side.

Now I succeeded, indeed, in observing the expected phenomena (doublet and triplet $a$ ).

4. For observing the doublet along the lines of force a perforated pole was used. With a current of $30 \mathrm{amp}$, the distance of the poles being as small as possible without interfering with the cadmium electrodes, a perfectly defined doublet was seen. One of the components appeared to be left-handed, the other right-handed circularly polarized $\dagger$ over the whole width. It might still be argued that the intervening dark space between the components is caused by a reversal and that it is not a doubling of the lines. However, then it must be supposed that this absorption-line just intercepts that part of a magnetically broadened line emitting unpolarized light. Now with a smaller value of the magnetic force the dark space becomes narrower. I grant that this is also the case with the supposed absorption-line. However, then a greater part of the broadened emission-line should emit unpolarized light, the smaller absorption-line now intercepting but a part of it. It appeared, however, that even with very narrow intervening dark space, the components of the doublet were entirely circularly polarized, and hence the intervening dark space seems to be different from an absorption-line. There is yet another argument against the

* C. R. 1897, t. cxxiv. pp. $748 \& 242$.

$\dagger$ The sign of the circular polarization is the same in the case of this cadmium line and of the D-lines. I must, however, correct my statement in $\$ 24$ of my former paper. I now see that if the lines of force are running towards the grating, the right-handed circularly polarized rays appear to have the greater period. Hence the radiation is due chiefly to the motion of a negatively-charged particle. Probably my mistake arose from a faulty indication of the axis of the $\lambda / 4$-plate used. 
interpretation that the dark space is of the nature of an absorption-band. Indeed, one should expect to see also reversal if looking across the lines of force. However, in this direction no trace of an ordinary absorption-line was visible with the same magnetic intensity. Hence we must conclude, I think, that I have really observed the doublet indicated by Lorentz's theorv.

10. Looking across the lines of force I succeeded in observing" the phenomenon indicated as triplet $a(\$ 6$, diagram). If no nicol was used only a broadened line was visible. A nicol oriented so as to get rid of light whose plane of polarization is vertical makes a dark well-defined line appear in the midst of the broadened line. Turning the nicol over $90^{\circ}$ this dark line disappears, the lateral wings now being quenched, because these are polarized in a horizontal plane. All this is to be expected according to $\$ 6$. The analysers in $\$ \S 9$ and 10 were inserted according to Lodge's * advice before the light reaches the grating.

Till now I have not had the opportunity of measuring the intensity of the field used in $\$ \$ 9$ and 10 . I took care, however, to make them nearly equal in the two cases.

11. Finally it may be mentioned that I observed also traces of the phenomena described in $\$ \S g$ and 10 with sodium. As the D-lines reverse so very easily by nonmagnetic agency and as they show sometimes complicated phenomena (Lockyer and Ciamician $\dagger$ ), it seems difficult to see the phenomena pure and simple with sodium. It is true that it is not difficult to avoid perturbations caused by the motion of the piece of asbestos, as mentioned by Egoroff and Georgiewsky $\ddagger$ in their interesting note. To avoid disturbances of this kind I simply moistened the mouth-piece of the burner with a very concentrated solation of $\mathrm{NaCl}$ or $\mathrm{NaBr}$. Small disturbances nevertheless easily make their appearance.

12. Although my inquiry is not yet closed, I think, however, that we can say that it seems to give new evidence in favour of the interpretation of the magnetization of the spectral lines given by Lorentz's theory. When I have determined the intensity of my field, we can decide the question whether we can hope to obtain a pure triplet by augmentation of the magnetic force or by using narrower spectral lines, though, of course, it may yet turn out that the phenomena prove to be less simple. For a future paper also inust be reserved the accurate measurement of the amount of the magnetic change

* 'The Electrician' of 26 February, 1897.

$\dagger$ cf. Kayser, Spectralanalyse, p. 305.

† C. R. t. cxxiv. p. 949 . 
with $\mathrm{Cd}$ and other substances *, and therewith the discussion about the ratio between mass and charge of the ions in Lorentz's theory. It is very probable that these "ions" differ from the electrolytical. It is true that by means of the latter many phenomena can be interpreted, as also is done in a paper by Richarz $\dagger$, where, e. g., the molecular magnetism is explained by the motion of the ions, but the high value of $\mathrm{e} / \mathrm{m}$ which $I$ have found makes it extremely improbable that we have to deal with the same mass in the two cases. And Lodge $\ddagger$ has even pointed out in an extremely interesting paper that my value of $e / m$ can be explained without the necessary motion of any "matter" at all.

Appendix.-On communicating the observations of this paper to Prof. Lodge, he most kindly informed me that he had already seen some of them himself. Especially he had seen the doubling of the lines and shown them at the Royal Society soirée on May 20. He, however, did not suppose it to be a real doubling of the line, but thought it to be a broadening and a reversal. I have given my reasons (the chief being the circular polarization over the whole width) for thinking that I observed a real doubling. I am confirmed in this opinion because it corroborates the observation of the perpendicular polarizations of the middle and of the edges of the triplet $a$ $(\$ 10)$. As Prof. Lodge does not mention that he observed this last phenomenon, and as my point of view is different from his, his object not being in the first place to test Lorentz's theory, I publish the paper in its original form, though it turns out that part of its contents has been observed by both of us independently $\S$.

Amsterdam, June 4.

* I have experiments in progress (measurements of the photographed magnetized spectrum) by which I hope to be able to obtain fairly accurate values of the magnetic change.

$\dagger$ Wied. Ann. lii. p. 385 (1894).

$\ddagger$ 'The Electrician,' Mar. 12, 1897.

$\S$ Disclaimer by Prof. Lodat.

Having been asked to exhibit Prof. Zeeman's discovery at the Royal Society soirée I arranged apparatus to obtain it more powerfully, and thereby saw the new effects; but without any intention of trespassing on the prerogative of the discoverer.

What I saw concerning polarization was that, when looking across the lines of force, a nicol brougbt out the doubling (or tripling or quadrupling as the case might be) more sharply, no doubt by quenching the residual light; and that it restored the original appearance of the line when rotated through $90^{\circ}$. I mention this here as partially confirming. Prof. Zeeman's most interesting and much more complete investigation, on the theoretical aspect of which at present I offer no further opinion.-Liverpool, June 6. 